\title{
Central Sleep Apnoea in Children
}

\author{
Md Atiar Rahman ${ }^{1 *}$, Selina Khanum ${ }^{2}$ and ARM Lutful Kabir ${ }^{3}$ \\ ${ }^{1}$ Associate Professor of Pediatric Pulmonology, Department of Pediatrics, Bangabandhu Sheikh Mujib Medical University, Dhaka. Bangladesh. \\ ${ }^{2}$ Professor of Pediatric Pulmonology and Chairman Department of Pediatrics, Bangabandhu Sheikh Mujib Medical University, Dhaka. Bangladesh. \\ ${ }^{3}$ Professor and Head of Department of Pediatrics, AD-Din Women Medical College, Boro Moghbazar, Dhaka. Bangladesh.
}

\begin{abstract}
Objectives: Central sleep apnoea (CSA) has been described in the adult population but there is limited information in children. So, the objective of this study was to focus epidemiology, clinical manifestations, pathophysiology, investigation and proper management of CSA in children.

Methods: By performing a search in PubMed, Cochrane Library and EMBASE, GOOGLE with the keywords: (central sleep apnoea* children*) AND Literature searches were performed in order to identify material relating central sleep apnoea in children since 1980 to 2020 . Relevant articles related to central sleep apnoea were identified and taken as a reference paper for preparing this article. A literature search was carried out based on a predefined series of key clinical questions. and the strategies included filters to limit the results by study type (reviews, randomized controlled trials and other types of experimental research) and age range (0-18 yrs.). In all cases, the results were limited to English language material.

Result: Central sleep apnoea (CSA) is a disorder in which there is recurrent cessation of breathing while asleep during night-time. These night-time breathing disturbances can lead to cardiovascular and neurological disorder. There are several clinical presentations of CSA, including periodic breathing, apnoea, snoring, gasping, hyperactivity restless sleep, daytime sleepiness. Abnormal ventilator function during sleep is the key point of CSA. The prevalence of Central sleep apnoea (CSA) in healthy children is thought to be about 4-6\%. The classification of CSA can be depending on the hypercapnia or no hypercapnia based on CO2 level in wakefulness stage and physiologic, idiopathic, or secondary CSA with the underlying diseases condition. Whole night polysomnography is the gold standard test for diagnosis of central sleep apnoea in children. Other investigation depends on the underlying causes. The management of CSA include counselling, oxygen supplementation, medication, non-invasive ventilation, and surgical intervention. After screening high risk children will undergo earlier diagnosis and timely therapeutic interventions.

Conclusion: Similar to adult patients, children with CSA present with complaints of insomnia, daytime sleepiness, and sometimes symptoms of obstructive sleep apnoea. In healthy children CSA is rare but if happened need extensive investigation and to find out exact underlying condition and also offer treatment accordingly.
\end{abstract}

\section{Introduction}

Central sleep apnoea (CSA) may be defined as there is recurrent cessation and resumption of breathing 20 or more second, at least two breaths leads to sleep fragmentation, ultimately lead to excessive daytime sleepiness, frequent nocturnal awakenings. It is the part of Paediatric sleep-disordered breathing (SDB), a general term for breathing difficulties during sleep. SDB can range from frequent loud snoring to obstructive sleep apnoea (OSA). CSA which may be physiologic, idiopathic (primary) and secondary. CSA is common in preterm babies and continues to present during infancy but rare afterwards in children. Children with idiopathic central sleep apnoea is non hypercapnic but have central apnoea during sleep, with normocapnia or hypocapnia during wakefulness without any underlying medical illness [1]. This can often be very difficult to separate from the normal individual children. The basic mechanism is that respiratory center does not inmate an attempt to breathe during these pauses. A few numbers of apneic pauses are normally found in all infants, children and young people. A child with CSA found longer and more frequent apnoea. CSA usually found when the brain does not send the proper nervous signals to the responsible muscles that control breathing [1-3].

The CCHS (Congenital Central Hypoventilation Syndrome) is another rare disorder in children usually present from birth and is characterized by alveolar hypoventilation without evidence of lung, neuromuscular or structural brainstem abnormalities. The disorder is due to mutations in the PHOX2b gene.

The prevalence of Central sleep apnoea in newborns less than 29 weeks' gestation is about $25 \%$. In healthy children, short duration $(<20$ s) central sleep apnoea is normal in terms of sigh, movement. The central sleep apnoea is gradually decreasing in nature over the time due to maturation of brainstem, after two years it is rare. The overall prevalence of CSA in children is about 4 to $6 \%$ [4-7].

\section{Data extraction}

By performing a searched in Pub Med, the Cochrane Library and EMBASE, GOOGLE with the keywords: (central sleep apnoea* children $^{\star}$ ) AND Literature searches were performed in order to identify material relating central sleep apnoea in children since 1980 to 2020 on a predefined series of key clinical questions and the strategies included filters to limit the results by study type (reviews, randomized controlled trials and other types of experimental research) and age

*Correspondence to: Md Atiar Rahman, Associate Professor of Pediatric Pulmonology, Department of Pediatrics, Bangabandhu Sheikh Mujib Medical University, Dhaka. Bangladesh, E-mail: atiar777@yahoo.com

Key words: children, central sleep apnoea

Received: August 12, 2020; Accepted: August 24, 2020; Published: August 31, 2020 
range $(0-18$ yrs.) From predefined questionnaire relevant articles related to central sleep apnoea were identified and taken as a reference paper for preparing this article. In all cases, the results were limited to English language material.

\section{Result}

\section{Definition and epidemiology}

Central sleep apnoea (CSA) may be defined as there is recurrent cessation and resumption of respiration 20 or more second, leads to sleep fragmentation, ultimately lead to excessive daytime sleepiness, frequent nocturnal awakenings, or both. It is the part of Paediatric sleep-disordered breathing (SDB), may be described as breathing difficulties during sleep. SDB can range from frequent loud snoring to obstructive sleep apnoea (OSA). CSA which may be Physiologic, idiopathic (primary) and secondary. [1]

The prevalence of Central sleep apnoea in preterm newborn less than 29 weeks' gestation is almost $25 \%$. In healthy children, short duration $(<20 \mathrm{~s})$ central sleep apnoea is normal in terms of sigh, movement. The central sleep apnoea is gradually disappearing over the time due to maturation of brainstem, after two years it is rare. The overall prevalence of CSA in children is about 4 to $6 \%$ [4-7].

Polysomnography finding in central sleep apnoea in children is very important. A finding of 5 central events per hour is considered clinically significant [8-11]. But if number is less causing a specific disorder or syndrome. In fact, there is no threshold level or number in central apnoea associated with disease. Normal data of, polysomnographic recordings in healthy term infants estimate the median Central Apnoea Index (CAI) at one month of life to vary between $5.5 / \mathrm{h}$ and $8.8 / \mathrm{h}$ with a duration ranging between 3.1 and $20.1 \mathrm{~s}$ when CA was a central pause lasting for greater than $3 \mathrm{~s}$ [12-16]. Central apnoea usually reduced by the second and third month of life and continue up to second year of life [12-16]. Beyond this age Marcus et al found that in otherwise healthy $1-18$ year olds $(n=50)$, the central apnoea lasted between 10 and $18 \mathrm{~s}$ was $30 \%$ and CAI is significant when it is more than 1 per hour in another study [14-16]. Normative data from several studies across North America and Europe showed that CAI is not related to desaturation, though 3 or more percent desaturation occur in central sleep apnoea.

\section{Pathophysiology}

The Brainstem, the control system of breathing can be affected by a number of conditions that ultimately lead to CSA. Blood carbon dioxide (CO2) level is influencing the brainstem to breath either in hyper or hypoventilation. [2,3,7]

Ventilation or breathing is controlled mainly at peripheral level means at the carotid body by $\mathrm{PaO} 2$ and $\mathrm{PaCO} 2$ ) and in center means the brainstem in the medulla by $\mathrm{CO} 2$ via shifts in $\mathrm{H}+$ concentration in the blood. This $\mathrm{PaO} 2$ or $\mathrm{PaCO} 2$ ("chemo sensitivity"), $\mathrm{CO} 2$ level in blood vary individual and with comorbid or diseases condition. This chemo response an individual at risk for unstable breathing patterns because "over respond" or under response to chemical stimuli. This $\mathrm{PaCO} 2$ have the negative and positive feedback response, when the level is decreased then hypoventilation and level increased hyperventilation, in this way breathing rhythm is maintained. Prolonged hyperventilation leads to reduced cardiac output and apnoea. Just as high chemo sensitivity can be destabilizing to the respiratory system; severely blunted chemo sensitivity can also be deleterious to cardiorespiratory homeostasis because extremely severe blood gas disturbances occur before a response is mounted. [4-7]

Normative data for central apnoea at different ages is sparse, and there is potential for wide variation within probable normal limits, particularly in the first few year of life.

Idiopathic central sleep apnoea in general, including in adults, is relatively poorly understood, and the approach is often excluding potential serious causes and then supporting management is pragmatically given. One of the better series of normative polysomnographic (PSG) data in the paediatric population [6-9] looks at detailed PSG in 209 healthy German children aged between 1 and 18 years. The absolute numbers in each age band are still relatively small; however, this gives a rough guide to inform assessment of potential significance of central apnoea events.

This data suggests central events are gradually reduced with time and relatively rare once out of infancy, but they do still occur, and the range of normal - 10th-90th centiles - can be quite significant, even for relatively older children [6-8] (Table 1).

Central apnoea/hypopneas are seen physiologically in healthy children but duration less than 20 second following arousal events in sleep (post-sigh), and also at transitions between different stages of sleep, as the control of breathing alters depending on sleep stage. For each individual there is a level of partial pressure of carbon dioxide the apnoea threshold - where if pCO2 falls below this level, an apnoea will be reflexively triggered. The apnoea threshold is usually 3-6 $\mathrm{mmHg}$ below physiological normal levels, but for some individuals may be as little as 1-2 mmHg below the normal range and the apnoea threshold is adjusted at different stages of sleep.

During the transitional period between wake and sleep, the 'wake' component of respiratory drive falls away, which can result in an increased observation of central events at sleep onset. If children have increased arousals to wake, or wakenings through the night, then the number of these transition events may be increased.

Central events are also seen physiologically at high altitude due to hypoxia, where relative hyperventilation will lower the partial pressure of carbon dioxide below the apnoeic threshold [7-9]. Neonates and infants classically have periodic breathing with increased central events which usually improve over the first year of life, as respiratory centre in brainstem matures. Older children may have persistence of this pattern with normal neurodevelopmental progress and examination finding is normal and no other identified problems (including no daytime symptoms suggestive of sleep fragmentation and no significant effect on gas exchange), in which case we would expect this will continue and improve with time, and an expectant/supportive management approach is often appropriate. For these children repetition of sleep studies at 6-12 months are often indicated.

But when there have been found an unexpectedly high number of events, trajectory is not improving, there are reported symptoms related to central sleep apnoea, or where there is a significant effect on gas exchange, then investigation and management are more likely to be indicated. There is an important distinction between central

Table 1. Shows central apnoea/hour in 1 to 18 -year-old children between 10th -90th centiles

\begin{tabular}{|l|c|c|c|c|c|c|c|c|}
\hline Age (yr) & 1.4 & 3.0 & 5.0 & 8.0 & 11.5 & 12.5 & 15.2 & 16.9 \\
\hline $\begin{array}{l}\text { Central apnoea/hr } \\
\text { (median) }\end{array}$ & 2.8 & 1.5 & 1.1 & 0.9 & 0.4 & 0.5 & 0.1 & 0.1 \\
\hline $\mathbf{1 0}^{\text {th }}$ centile & 1.0 & 0.7 & 0.5 & 0.3 & 0 & 0.1 & 0.1 & 0.1 \\
\hline $\mathbf{9 0}^{\text {th }}$ centile & 4.3 & 6.9 & 3.2 & 2.7 & 1.2 & 1.8 & 0.8 & 1.3 \\
\hline
\end{tabular}


sleep apnoea (pauses in breathing with a non-obstructive aetiology) and central hypoventilation (under breathing in sleep resulting in demonstrable impairment of carbon dioxide clearance with a nonobstructive aetiology). The two may be found together but can also occur independently.

\section{Causes of central sleep apnoea}

CSA which may be idiopathic (primary) and secondary. The Secondary CSA has various causes [7-9].

In newborn and infancy prolonged apnoeic episode occur due to immature brainstem. Or other congenital abnormalities affecting the nervous system. Global developmental delay is sometimes associated with an increased likelihood of persistence of 'normal' central events.

- Prader-Willi Syndrome

- Central Nervous System (CNS) inflammation/infection

- Structural brainstem lesions or malformations

- Achondroplasia (or other conditions which affect craniofacial structure)

- Neurometabolic disorders

- Shy-Drager Syndrome, familial dysautonomia

- Spinal injury

- Obesity hypoventilation syndrome

- Congenital Central Hypoventilation Syndrome

- Gastro-oesophageal reflux diseases.

- Epilepsy

- NIV-triggered central events

- Obstructive Sleep Apnoea may act as a trigger, and obstructive and central events often co-exist in obstructive sleep apenia (OSA)

- Neuromuscular disorders

- Idiopathic

- Down syndrome

- Co morbid conditions in the ICSA group included attention deficit hyperactivity disorder, bipolar mood disorder, anxiety, depression, Asperger syndrome, hypotonia, rheumatoid arthritis, Down syndrome, obesity, asthma, allergic rhinitis, eczema and gastro oesophageal reflux. [1]

\section{Common signs and symptoms of CSA include [7-9]}

- Episodes of stopped breathing or abnormal breathing patterns during sleep

- Abrupt awakenings with shortness of breath

- Difficulty in staying asleep (insomnia)

- Excessive daytime sleepiness (hypersomnia)

- Difficulty in concentrating capacity

- Mood changes

- Morning headaches

- Snoring

\section{Investigation}

\section{Initial approach to investigation is as follows}

Whole night polysomnography is the gold standard test for diagnosis of central sleep apnoea in children [7-10]. A finding of 5 central events per hour is considered clinically significant [8-11]. But if number is less causing a specific disorder or syndrome. In fact, there is no threshold level or number in central apnoea associated with disease. Detailed general examination, including consideration of neurodevelopment assessment is important if there is clear hypoventilation or a very suggestive clinical picture, then Congenital Central Hypoventilation Syndrome (CCHS) should be considered and genetic investigations like Paired-like home box $2 \mathrm{~b}$, a protein that in humans is encoded by the PHOX2B gene located on chromosome 4 (PHOX2B gene) will be adviced.

It is important to be aware that PHOX2B testing is not available in developing countries but available in developed countries like the United Kingdom (UK), United State (USA) and some of the European countries. In fact, it does NOT absolutely exclude the diagnosis and it is essential that the whole clinical picture is considered before its advice. It should not be advised to every child with central respiratory events. Before PHOX2B testing, it is needed to consult with respiratory team. Full sequence PHOX2B analysis is only available in Chicago. [7-9,16]

\section{Infant with central episodes [7-9]}

- Oesophageal PH monitoring for reflux disease

- Genetics: karyotype +/- Prader-Willi syndrome

- Sleep electroencephalogram (EEG) discuss with neurology

- Neurometabolic screening

- Magnetic resonance imaging (MRI) (+/- MRI Brain)

- Consider endocrine assessment/investigation

- Echocardiogram

Older child with central episodes [7-9]

- Genetics: karyotype +/- Prader-Willi

- MRI Brain (with specific attention to the brainstem - mainly looking for Chiari malformations, though most usually normal)

- Sleep electroencephalogram (EEG) discuss with neurology.

- Investigation for neurometabolic disorders

- Echocardiogram

- Oesophageal PH monitoring for reflux disease

- Endocrine evaluation

If strong clinical suspicion of CCHS [7-9]

- $\mathrm{PHOX} 2 \mathrm{~B}$

- Computed tomography scan (CT) Chest/Abdomen

- Metaiodobenzylguanidine (MIBG)

- Electrocardiogram (ECG) + 72-hour Holter recording 


\section{Management}

Management is coordinated by the initial clinician with the help of Paediatric Neurologist, Respiratory team and Sleep team, it may be more appropriate to transfer primary care to the sleep centre for proper care. Individual cases, particularly review of diagnostic sleep studies, can always be considered with the discussion with sleep consultant. Treatment depends on underlying cause and supportive measures also very important for CSA patient.

If all investigations are normal, then a diagnosis of idiopathic central sleep apnoea is usually appropriate. Particularly in younger children, there is a good chance that this may represent a normal degree of developmental difference related to overall maturation of respiratory control, and there is therefore a reasonably good expectation of improvement with time. The overall strategy for management is explanation, reassurance, exclusion of potentially significant causes of central respiratory events, and also to explain the concept of idiopathic central sleep apnoea (with the implication that we do not have a full understanding of the pathogenesis of this condition) and a pragmatic approach is also offered. Decision to treat rests on is depend on severity of underlying central episodes, degree of possible/actual documented effect on daytime symptoms, overall neurodevelopment progress, and clinician or parental preferences.

There is some evidence - although evidence in all aspects of this condition are limited - that children with idiopathic central sleep apnoea may have increased night awakenings, and increased daytime sleepiness. This can often be very difficult to separate from the normal individual children. Active management of CSA is principally supportive - either nocturnal oxygen or non-invasive ventilation depending on the clinical picture and underlying cause. [17]

Oxygen would be the preferred first line treatment, as usually simpler to organise and better tolerated. It both reduces the impact of desaturations from central event and has a stabilising effect on respiratory control, overall results in a reduction of the number of central episodes. There is some evidence from adults that while treating with low-flow oxygen can directly reduce the number of central events, but may precipitate obstructive events - these needs to be carefully monitored following initiation with oxygenation, leading to potential secondary hypoventilation. [17-18]

NIV would usually only be indicated for significant disruption, or where hypoventilation is an issue either at diagnosis, or noted at oxygen therapy initiation [8-9]. Bi-level positive airway pressure (BPAP) and Continuous Positive Airway Pressure (CPAP) also help in sleeping time to prevent apnoeic attack [7-10].

Use of medication to alter the apnoea threshold (e.g. acetazolamide) should be considered in specific situations, and after discussion with a respiratory consultant. Apnoea in newborn and infancy may improve over the time by maturing brainstem. These cases should be following up by a Sleep Consultant on time. Oxygen supplementation during sleep may reduce the number of apnoeic episodes pauses and reduced the number of CSA.

There is some medication that stimulate respiratory center in brainstem and reduce apneic attack [19,20]. It has been found that acetazolamide and theophylline improve CSA in patients with heart failure [21,22]. Acetazolamide and has also been shown to improve sleep disordered breathing (SDB) in ICSA [23]. Acetazolamide, carbonic anhydrase inhibitor causes metabolic acidosis that likely shifts the hypercapnic ventilatory response and lowers the $\mathrm{PaCO} 2$ apnoea threshold level in blood $[21,24,25]$. Theophylline can improve
SDB via increasing central respiratory drive and cardiac contractility. Though respiratory stimulants help in sleep apnoea, but it cannot be routinely recommended for CSA treatment at this time. Theophylline may increase the risk of cardiac arrhythmias and sudden death in apnoeic patients, $[17,26,27]$ and long-term trials exploring the efficacy and safety of acetazolamide is not yet available.

\section{Surgical}

Sometime brainstem is affected by skull or Arnold -Chiari malformation, and then surgery is the options for treatment.

\section{Summary and recommendations}

In healthy children CSA is rare but if happened need extensive investigation and to find out exact underlying condition, most common of which may be neuroanatomical abnormalities and remain asymptomatic long time.

\section{References}

1. Gurbani N, Verhulst SL, Tan C, Simakajornboon N (2017) Sleep complaints and sleep architecture in children with idiopathic central sleep apnea. J Clin Sleep Med 13: 777783. [Crossref]

2. AASM (2014) Central Sleep Apnea Syndromes. International Classification of Sleep Disorders. Darien, IL: American Academy of Sleep Medicine, 69-107.

3. Bounhoure JP, Galinier M, Didier A, Leophonte P (2005) Sleep apnea syndromes and cardiovascular disease. Bull Acad Natl Med 189: 445-459. [Crossref]

4. Bradley TD, McNicholas WT, Rutherford R, Popkin J, Zamel N, et al. (1986) Clinical and physiologic heterogeneity of the central sleep apnea syndrome. Am Rev Respir Dis 134: 217-221 [[Crossref]

5. Carroll JL, Donnelly DF (2014) Respiratory physiology and pathophysiology during sleep. In: Sheldon SH, Ferber R, Krieger M, Gozal D, editors. Principles and Practice of Paediatric Sleep Medicine.

6. White DP (2005) Pathogenesis of obstructive and central sleep apnea. Am J Respir Crit Care Med 172: 1363-1370. [Crossref]

7. McLaren AT, Bin-Hasan S, Narang I (2019) Diagnosis, management and pathophysiology of central sleep apnea in children. Paediatr Respir Rev 30: 49-57. [Crossref]

8. Scholle S, Wiater A, Scholle HC (2012) Normative values of polysomnographic parameters in childhood and adolescence: arousal events. Sleep Med 13: 243-251. [Crossref]

9. Scholle S, Beyer U, Bernhard M, Eichholz S, Erler T, et al. (2011) Normative values of polysomnographic parameters in childhood and adolescence: quantitative sleep parameters. Sleep Med 12: 542-549. [Crossref]

10. Berry R, Brooks R, Garnaldo C, Hardig S, Lloyd R, et al. (2017) The AASM Manual for the scoring of sleep and associated events: rules, terminology and Technical specifications

11. Kritzinger FE, Al-Saleh S, Narang I (2011) Descriptive analysis of central sleep apnea in childhood at a single center. Pediatr Pulmonol 46: 1023-1030. [Crossref]

12. Brockmann PE, Poets A, Poets CF (2013) Reference values for respiratory events in Overnight polygraphy from infants aged 1 and 3 months. Sleep Med 14 :1323-1327. [Crossref]

13. Schlüter B, Buschatz D, Trowitzsch E (2001) Polysomnographic reference curves for the first and second year of life. Somnologie 5: 3-16.

14. Marcus CL, Omlin KJ, Basinki DJ, Bailey SL, Rachal AB, et al. (1992) Normal polysomnographic values for children and adolescents. Am Rev Respir Dis 146: 12351239. [Crossref]

15. Scholle S, Wiater A, Scholle HC (2011) Normative values of polysomnographic Parameters in childhood and adolescence: cardiorespiratory parameters. Sleep Med 12: 988-996. [Crossref]

16. Montgomery-Downs HE, O’Brien LM, Gulliver TE, Gozal D (2006) Polysomnographic Characteristics in normal preschool and early school-aged children. Pediatrics 117 741-753. [Crossref]

17. White DP, Gleeson K, Pickett CK, Rannels AM, Cymerman A, et al. (1987) Altitude acclimatization: influence on periodic breathing and chemo responsiveness during sleep. J Appl Physiol 63: 401-412. [Crossref] 
18. Poulain M, Doucet M, Major GC, Drapeau V, Sériès F, et al. (2006) The effect of obesity on chronic respiratory diseases: pathophysiology and therapeutic strategies. Can Med Assoc J 174:1293-1299. [Crossref]

19. Don DM, Geller KA, Koempel JA, Ward SD (2009) Age specific differences in pediatric obstructive sleep apnea. Int J Pediatr Otorhinolaryngol 73: 1025-1028. [Crossref]

20. McNicholas WT, Carter JL, Rutherford R, Zamelb N, Phillipson EA (1982) Beneficial effect of oxygen in primary alveolar hypoventilation with central sleep apnea. Am Rev Respir Dis 125: 773-775 [Crossref]

21. Sutton FD Jr, Zwillich CW, Creagh CE, Pierson DJ, Weil JV (1975) Progesterone for outpatient treatment of Pickwickian syndrome. Ann Intern Med 83: 476-479. [Crossref]

22. Javaheri S, Parker TJ, Wexler L, Liming JD, Lindower P, et al. (1996) Effect of theophylline on sleep-disordered breathing in heart failure. $N$ Engl J Med 335:562-567. [Crossref]
23. Avaheri S (2006) Acetazolamide improves central sleep apnea in heart failure: a double-blind, prospective study. Am J Respir Crit Care Med 173: 234-237. [Crossref]

24. Simantirakis EN, Schiza SE, Chrysostomakis SI, Chlouverakis GI, Klapsinos NC, et al (2005) Atrial overdrive pacing for the obstructive sleep apnea-hypopnea syndrome. $N$ Engl J Med 353: 2568-2577. [Crossref]

25. White DP, Zwillich CW, Pickett CK, Douglas NJ, Findley LJ, et al. (1982) Central sleep apnea: improvement with acetazolamide therapy. Arch Intern Med 142:18161819. [Crossref]

26. Nakayama H, Smith CA, Rodman JR, Skatrud JB, Dempsey JA (2002) Effect of ventilatory drive on carbon dioxide sensitivity below eupnea during sleep. Am J Respir Crit Care Med 165:1251-1260. [Crossref]

27. Bittar G, Friedman HS (1991) The arrhythmogenicity of theophylline: a multivariate analysis of clinical determinants. Chest 99:1415-1420. [Crossref]

Copyright: @2020 Rahman MA. This is an open-access article distributed under the terms of the Creative Commons Attribution License, which permits unrestricted use, distribution, and reproduction in any medium, provided the original author and source are credited. 